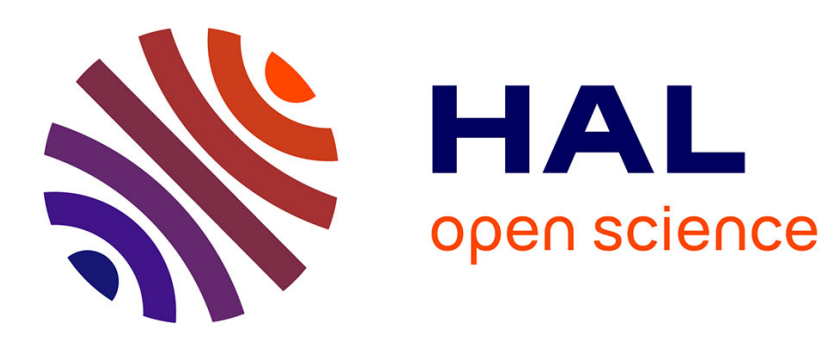

\title{
Mobilité atomique et défauts ponctuels dans des composés intermétalliques à base de $\mathrm{Ni3Al}$ et de TiAl
}

O. Dimitrov, T. Tarfa, B. Sitaud, C. Dimitrov

\section{To cite this version:}

O. Dimitrov, T. Tarfa, B. Sitaud, C. Dimitrov. Mobilité atomique et défauts ponctuels dans des composés intermétalliques à base de Ni3Al et de TiAl. Journal de Physique IV Proceedings, 1996, 06 (C2), pp.C2-79-C2-88. 10.1051/jp4:1996211 . jpa-00254189

\section{HAL Id: jpa-00254189 https://hal.science/jpa-00254189}

Submitted on 1 Jan 1996

HAL is a multi-disciplinary open access archive for the deposit and dissemination of scientific research documents, whether they are published or not. The documents may come from teaching and research institutions in France or abroad, or from public or private research centers.
L'archive ouverte pluridisciplinaire HAL, est destinée au dépôt et à la diffusion de documents scientifiques de niveau recherche, publiés ou non, émanant des établissements d'enseignement et de recherche français ou étrangers, des laboratoires publics ou privés. 


\title{
Mobilité atomique et défauts ponctuels dans des composés intermétalliques à base de $\mathrm{Ni}_{3} \mathrm{Al}$ et de TiAl
}

\author{
O. Dimitrov, T. Tarfa, B. Sitaud et C. Dimitrov
}

Centre d'Etudes de Chimie Métallurgique, CNRS, 15 rue Georges Urbain, 94407 Vitry-sur-Seine cedex, France

\begin{abstract}
Résumé : Les composés intermétalliques, en particulier les aluminiures de nickel et de titane, ont des potentialités importantes comme matériaux de hautes températures. Un certain nombre de leurs caractéristiques (stabilité microstructurale, fluage,...) sont directement liées à un transport de matière et sont donc conditionnées par les propriétés des défauts lacunaires. Une détermination expérimentale de la mobilité atomique et des propriétés des lacunes dans ces composés est importante d'une part pour connaître la variation de ces caractéristiques en fonction de la composition chimique, d'autre part pour valider les modèles théoriques et leur fournir les coefficients numériques nécessaires pour prévoir les évolutions à long terme.

Dans le cas des matériaux basés sur le système $\mathrm{Ni}-\mathrm{Al}$, nous présenterons les données qui ont été obtenues sur des composés modèles très simples, destinés à représenter les matériaux intermétalliques de structure $\mathrm{Ll}_{2}$. Il s'agit de phases binaires Ni3Al et ternaires $\mathrm{Ni3}(\mathrm{Al}, \mathrm{Ti})$ ou $\mathrm{Ni3}(\mathrm{Al}, \mathrm{Ta})$, que nous comparerons à des solutions solides du même système. Deux séries d'études ont permis de caractériser d'une part la mobilité atomique globale (qui intègre les caractéristiques de formation et de migration des lacunes), en déterminant les cinétiques de relaxation de l'ordre atomique (ordre à courte distance ou ordre à grande distance), d'autre part l'une des composantes de cette mobilité : la vitesse de migration des lacunes, en étudiant l'élimination de lacunes introduites en excès. On montrera les différences de comportement entre les deux types de phases, et l'influence importante de la composition chimique (éléments majeurs et éléments d'addition) aussi bien dans les solutions solides que dans les composés intermétalliques.

Les méthodes employées dans les alliages à base $\mathrm{Ni}$ - $\mathrm{Al}$ sont applicables (et ont commencé à être appliquées) dans d'autres systèmes d'intérêt aéronautique, en particulier les aluminiures de titane.
\end{abstract}

\section{LES COMPOSES INTERMETALLIQUES COMME MATERIAUX POUR HAUTES TEMPERATURES : IMPORTANCE DU TRANSPORT DE MATIERE PAR DIFFUSION.}

Les matériaux destinés à des applications aux températures élevées ( par exemple systèmes de production d'énergie, applications aérospatiales, moteurs d'automobiles,...) doivent présenter un certain nombre de qualités telles que des propriétés mécaniques élevées aux hautes températures, une bonne résistance au fluage, une faible sensibilité à l'environnement. 
Le fluage à haute température implique essentiellement un transport de matière (par flux de lacunes, par montée de dislocations,...). Il est donc régi par la diffusion atomique. Par exemple, dans le fluage en loi puissance, la vitesse de déformation s'écrit [1]:

$$
\dot{\varepsilon}=\mathrm{D}(\mathrm{Gb} / \mathrm{kT})(\sigma / \mathrm{G})^{\mathrm{n}}
$$

où $\mathrm{D}$ est un coefficient de diffusion, $\mathrm{G}$ le module de cisaillement, $\mathrm{b}$ le vecteur de Burgers, $\mathrm{k}$ la constante de Boltzmann, $T$ la température, $\sigma$ la contrainte et $\mathrm{n}$ un exposant. On voit donc que, dans ce mécanisme, la vitesse de fluage est directement proportionelle au coefficient de diffusion : la déformation sera d'autant plus faible que la diffusion sera plus lente.

Par ailleurs, la structure des matériaux est habituellement optimisée pour obtenir les propriétés exigées par une application spécifique. En service, des modifications structurales peuvent se produire et il est nécessaire de les maîtriser, pour assurer la fiabilité et la sécurité du comportement à long terme du matériau. Ici encore, les propriétés de diffusion sont fondamentales. Un cas classique mettant en jeu la stabilité de la microstructure est celui de la coalescence de précipités d'une seconde phase dans une matrice. Dans le modèle développe par Lifichitz et Slyozov [2] et par Wagner [3], le cube du rayon moyen $\bar{R}$ des particules varie linéairement avec le temps de séjour en température, suivant une loi

$$
\overline{\mathrm{R}}^{3}-\overline{\mathrm{R}}_{0}^{3} \approx \mathrm{D}\left(\mathrm{C}_{0} \mathrm{~V} \gamma_{\mathrm{s}} / \mathrm{kT}\right) \mathbf{f t}
$$

où $\bar{R}_{0}$ est le rayon moyen des particules à l'instant initial, $\mathrm{C}_{0}$ la concentration de soluté dans la matrice, $\mathrm{V}$ le volume atomique de la phase précipitée, $\gamma_{s}$ l'énergie de l'interface précipité-matrice, $f$ une fonction faisant intervenir la fraction volumique de précipités et t le temps. L'accroissement de la taille des particules, donc l'éventuelle dégradation des propriétés mécaniques, sera d'autant plus rapide que le coefficient de diffusion de l'espèce atomique transportée sera plus grand. Un autre cas est celui des matériaux intermétalliques basés sur le composé TiAl $\gamma$. Leur microstructure est composé de fines lamelles de phase $\gamma$, avec une petite fraction volumique de lamelles $\alpha_{2}$, et conduit à un ensemble optimal de propriétés mécaniques; il est important que l'évolution de cette microstructure aux températures de service soit la plus faible possible.

Dans les matériaux métalliques soumis à des sollicitations thermiques (c'est-à-dire en l'absence d'irradiation) la diffusion atomique a lieu par un mécanisme lacunaire. Le coefficient de diffusion D est proportionnel (compte tenu d'un facteur de corrélation $\mathrm{f}$ ) à la fréquence des sauts d'atomes $v_{a t}$. Cette dernière varie comme le produit de la concentration de lacunes $\mathrm{C}_{\mathrm{L}}$ et de leur fréquence de saut $\mathrm{v}_{\mathrm{L}}$.

$$
\mathrm{D} \sim \mathrm{f} v_{\mathrm{at}} \approx \mathrm{C}_{\mathrm{L}} v_{\mathrm{L}}
$$

A l'équilibre thermodynamique, la concentration des lacunes dépend de la température suivant la relation

$$
\mathrm{C}_{\mathrm{L}}^{\mathrm{eq}}=\mathrm{A} \exp \left(-\mathrm{H}_{\mathrm{f}} / \mathrm{kT}\right)
$$

où $\mathrm{H}_{\mathrm{f}}$ est l'enthalpie de formation des lacunes.

Leur fréquence de saut s'écrit

$$
v_{L}=v_{L}^{0} \exp \left(-H_{m} / k T\right)
$$

où $v_{L}^{0}$ est la fréquence de vibration des atomes voisins d'une lacune, et $\mathrm{H}_{m}$ l'enthalpie de migration des lacunes.

En conditions isothermes, les lacunes sont à l'équilibre thermodynamique ; leur concentration et leur fréquence de saut sont entièrement déterminées par la température. La connaissance de

$$
v_{\text {at }} \approx C_{L}^{e q} v_{L}
$$

et de sa variation en température par l'intermédiaire de l'enthalpie d'autodiffusion, égale à

$$
\mathrm{H}_{\mathrm{D}}=\mathrm{H}_{\mathrm{f}}+\mathrm{H}_{\mathrm{m}}
$$

(sous réserve que f ne varie pas trop avec la température), sont suffisantes pour caractériser la mobilité atomique.

Par contre, dans des conditions de température variable, la concentration de lacunes à un instant donné peut être hors d'équilibre. Il faudra alors connaître séparément l'enthalpie de formation des lacunes $\mathrm{H}_{\mathrm{f}}$ et leur enthalpie de migration $\mathrm{H}_{\mathrm{m}}$ pour pouvoir analyser les cinétique d'évolution du matériau.

Les études expérimentales visant à caractériser la mobilité atomique ont donc deux objectifs :

1. Obtenir des informations sur la mobilité atomique, en déterminant la fréquence de saut atomique $v_{\mathrm{at}}$, sa variation en température et en déduire l'enthalpie d'activation $\mathrm{H}_{\mathrm{D}}$.

2. Obtenir des informations sur les composantes de cette mobilité, c'est-à-dire sur la fréquence de saut des 
lacunes et sur leur concentration, respectivement régies par l'enthalpie de migration des lacunes et (à l'équilibre) par leur enthalpie de formation.

\section{METHODES DE CARACTERISATION DE LA MOBILITE ATOMIQUE GLOBALE ET DES PROPRIETES DES LACUNES}

\subsection{Méthode de caractérisation de la mobilité atomique}

Le principe de la méthode utilisée repose sur la détermination de la cinétique de relaxation de l'ordre atomique homogène (dans les composés intermétalliques : Ordre à Grande Distance), à partir d'états proches de l'crire d'équilibre. Les évolutions étudiées ont toujours lieu entre des états d'ordre très proches, par changement des positions relatives d'atomes proches voisins. Il n'y a pas d'intervention de phénomènes de germination ou de croissance de domaines ordonnés.

Les variations de l'ordre atomique ont été suivies par des mesures de résistivité électrique résiduelle. La procédure utilisée consiste à:

- mettre le matériau à l'équilibre thermodynamique par recuit prolongé à une température $T_{1}$.

- imposer un petit changement de température $\Delta T$. A la nouvelle température $T_{2}=T_{1}+\Delta T$, l'alliage se trouve donc hors d'équilibre.

- suivre la relaxation isotherme de la résistivité résiduelle en fonction du temps jusqu'à ce qu'un nouvel état d'équilibre, correspondant à la température $T_{2}$, soit atteint.

La figure 1 montre l'évolution de la résistivité résiduelle dans $\mathrm{Ni}_{3} \mathrm{Al}$ stoechiométrique [4], au cours de tels recuits isothermes successifs, suivis d'une trempe destinée à retenir l'état structural réalisé à haute température. A chaque température de recuit, la résistivité évolue rapidement à partir de sa valeur initiale, puis atteint une valeur stationnaire pour les temps longs.

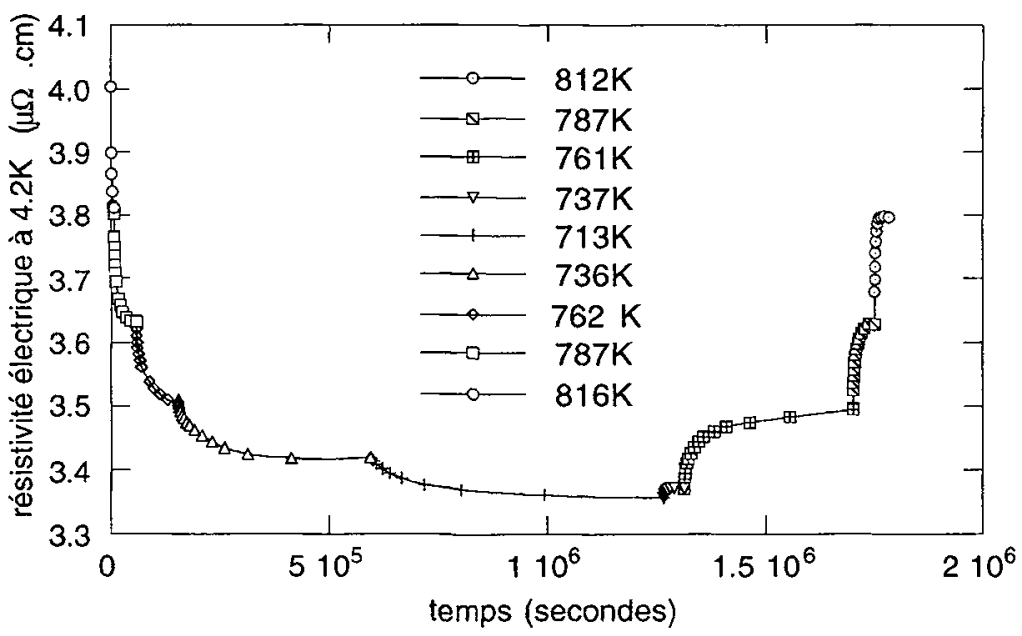

Figure 1. Evolution de la résistivité électrique résiduelle au cours de recuits isothermes successifs dans Ni3Al stoechiométrique [4].

Plusieurs informations sont tirées des courbes de ce type:

- les valeurs obtenues lors des recuits prolongés sont sensiblement indépendantes de la façon dont on approche l'équilibre (à partir d'états plus ordonnés ou d'états moins ordonnés). Elles renseignent sur l'état d'équilibre de 
l'ordre atomique et sur sa variation en fonction de la température [5].

- l'approche de l'équilibre fournit les lois de relaxation de l'ordre atomique, et les constantes de temps $\tau$ qui les caractérisent. Ces lois sont différentes dans les solutions solides [6] et dans les composés intermétalliques [7]. Dans la mesure où la relaxation met en jeu des échanges entre atomes voisins, l'inverse du temps de relaxation $\left(\tau^{-1}\right)$ est de l'ordre de la fréquence de saut atomique $v_{\mathrm{at}}$.

- la variation des constantes de temps en fonction de la température permet d'accéder à l'enthalpie d'activation $\mathrm{H}_{\mathrm{D}}$ régissant la mobilité atomique [5,8].

\subsection{Méthodes de caractérisation des propriétés des lacunes}

Pour caractériser les propriétés des lacunes. on introduit un faible excès de concentration de ces défauts mar irradiation électronique à basse température 19,10$]$. En élevant ensuite la température, les lacunes deviennent mobiles. migrent dans le résean de l'alliage, et fïnissent par s'éliminer par absorption dans des sites d'annihilation ou par formation d'aggrégats [11-13]. Au cours de ces processus, la variation de résistivité électrique résulte de deux types de mécanismes : d'une part les modifications de l'ordre atomique induites par la migration des lacunes, d'autre part la diminution progressive de la concentration de ces dernières. La seconde contribution est dominante dans les composés intermétalliques. Des mesures de résistivité résiduelle au cours de recuits consécutifs à une irradiation permettent donc de caractériser la mobilité des lacunes ; leur enthalpie de migration $\mathrm{H}_{\mathrm{m}}$ peut être obtenue en effectuant des sauts de température lors des recuits isothermes au cours desquels se produit la migration de ces défauts (méthode du changement de pente).

L'enthalpie de formation des lacunes peut alors être obtenue par différence entre ces valeurs et celles de l'enthalpie d'activation $\mathrm{H}_{\mathrm{D}}$ obtenues par les études de relaxation thermique de l'ordre atomique :

$$
\mathrm{H}_{\mathbf{f}}=\mathrm{H}_{\mathrm{D}}-\mathrm{H}_{\mathrm{m}} \text {. }
$$

\section{MATERIAUX ETUDIES : COMPOSES INTERMETALLIQUES SIMPLES A BASE DE NizAl ET DE TíAl}

Les matériaux sur lesquels ces déterminations ont été effectuées étaient des matériaux modèles les plus simples possibles (monophasés) susceptibles de représenter le comportement de composés intermétalliques intéressants pour des applications à hautes températures. Ces matériaux présentent soit la structure cristallographique $\mathrm{Ll}_{2}$, afin de modéliser les composés intermétalliques à base de $\mathrm{Ni} 3 \mathrm{Al}$, soit la structure $\mathrm{Ll}_{0}$ qui est celle de la phase principale des composés intermétalliques à base de Tỉ.

Ils ont été choisis pour mettre en évidence les effets de :

- la nature de l'ordre atomique,

- la composition chimique des matériaux (concentration en éléments majeurs, c'est-à-dire écarts à la stœchiométrie ; présence d'éléments d'addition).

Le tableau I présente les compositions des deux types de matériaux étudiés :

- composés intermétalliques binaires $\mathrm{Ni}_{3} \mathrm{Al}$ (stoechiométrique, sur-stoechiométrique ou sousstoechiométrique), ou ternaires (4\% d'aluminium ayant été substitués par $4 \%$ de titane ou de tantale).

- composés intermétalliques binaires TiAl (stoechiométrique ou sur-stoechiométriques).

Tableau I. Compositions des phases intermétalliques étudiées.

\begin{tabular}{|c|c|c|}
\hline & Phases $\mathrm{Ll}_{2}\left(\mathrm{Ni} 3 \mathrm{Al} \gamma^{\prime}\right)$ & Phases L10 (TiAl $\gamma)$ \\
\hline Binaires & $\begin{array}{l}24,6 \text { at\% Al } \\
\mathrm{Ni3} A l \text { avec } \\
25,0 \text { at\% Al } \\
26,5 \text { at\% Al }\end{array}$ & $\begin{array}{l}50,0 \text { at \% Al } \\
\text { à } \\
56,0 \text { at\% Al }\end{array}$ \\
\hline Ternaires & $\begin{array}{ll}\mathrm{Ni} 3(\mathrm{Al}, \mathrm{Ti}) & (21 \mathrm{at} \% \mathrm{Al}+4 \mathrm{at} \% \mathrm{Ti}) \\
\mathrm{Ni} 3(\mathrm{Al}, \mathrm{Ta}) & (21 \mathrm{at} \% \mathrm{Al}+4 \mathrm{at} \% \mathrm{Ta})\end{array}$ & \\
\hline
\end{tabular}




\section{MOBILITE ATOMIQUE ET PROPRIETES DES LACUNES DANS LES COMPGSES INTERMETALLIQUES Ni $\mathrm{Ni}_{3}$}

\subsection{Mobilité atomique globale}

Les vitesses de relaxation de l'ordre (qui sont déterminées par la fréquence des sauts atomiques) dans un certain nombre de phases $\gamma$ stoechiométriques, binaire ou ternaires, sont données sur la figure 2 en fonction de l'inverse de la température [8]. A titre de comparaison, on a également porté les vitesses de relaxation de l'ordre dans des solutions solides (phases $\gamma$ ) basées sur le même système Ni-Al. Les pentes de ces graphes permettent d'accéder aux valeurs de $\mathrm{HD}_{\mathrm{D}}$ (Tableau II).

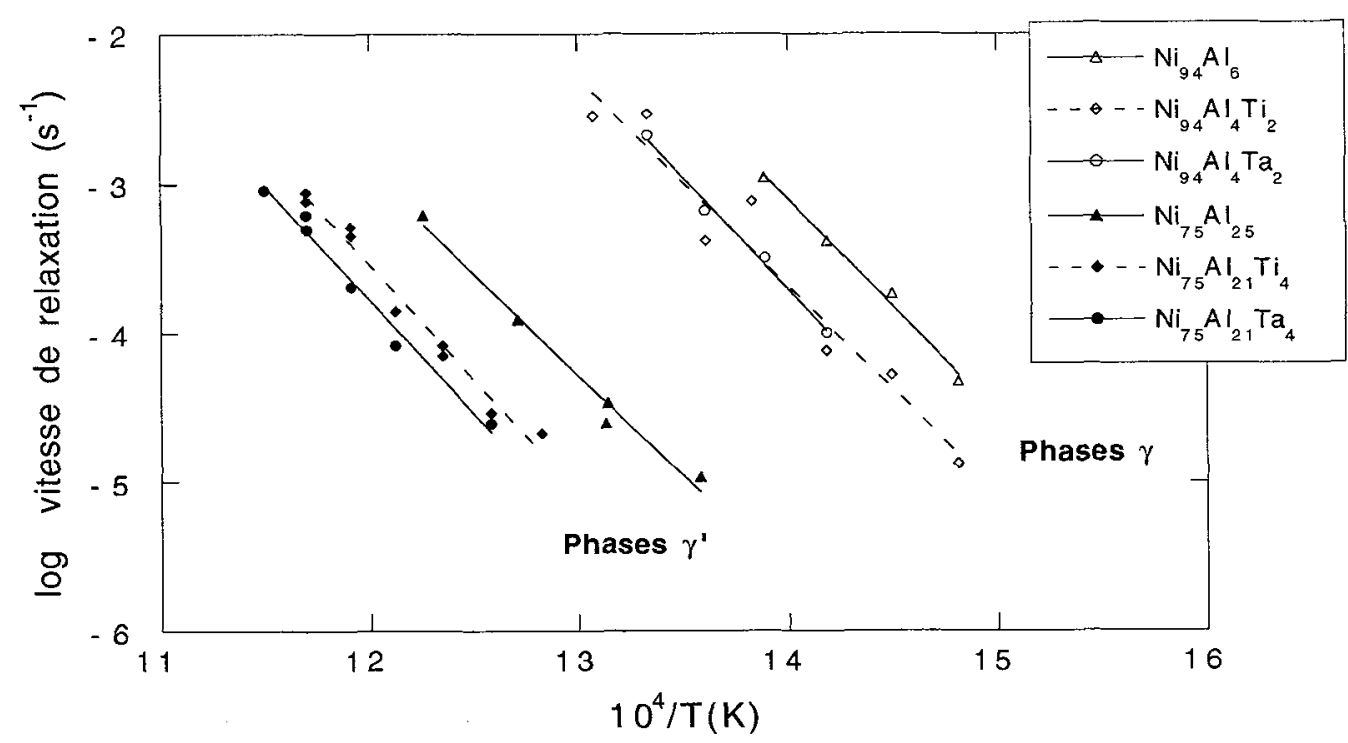

Figure 2. Influence de la température sur les vitesses de relaxation de l'ordre atomique dans des solutions solides et des composés intermétalliques basés sur le système $\mathrm{Ni}-\mathrm{Al}$ [8].

D'un point de vue général, on observe les tendances suivantes, pour ce qui concerne les vitesses de relaxation donc les mobilités atomiques :

- la mobilité atomique est beaucoup plus faible dans les phases $\gamma^{\prime}$ que dans les phases $\gamma$. Par exemple, vers $450^{\circ} \mathrm{C}$, la diffusion est 100 fois plus lente dans $\mathrm{Ni} 3 \mathrm{Al}$ que dans l'alliage $\mathrm{Ni}$ (Al 6). Cette différence semble surtout liée à un facteur pré-exponentiel plus faible dans $\gamma$.

- la présence des éléments Ti et Ta produit une diminution de la mobilité atomique aussi bien dans les phases $\gamma$ que dans les phases $\gamma[14,15]$. Dans ce dernier cas, l'effet de l'élément d'addition sur la mobilité atomique paraît être dû à par une augmentation de l'enthalpie d'activation.

- la mobilité atomique dépend de la teneur en éléments majeurs (figure 3). En particulier, dans les composés $\gamma$ binaires, elle est minimale à la composition stoechiométrique et augmente de part et d'autre de cette composition $[4,5]$. 


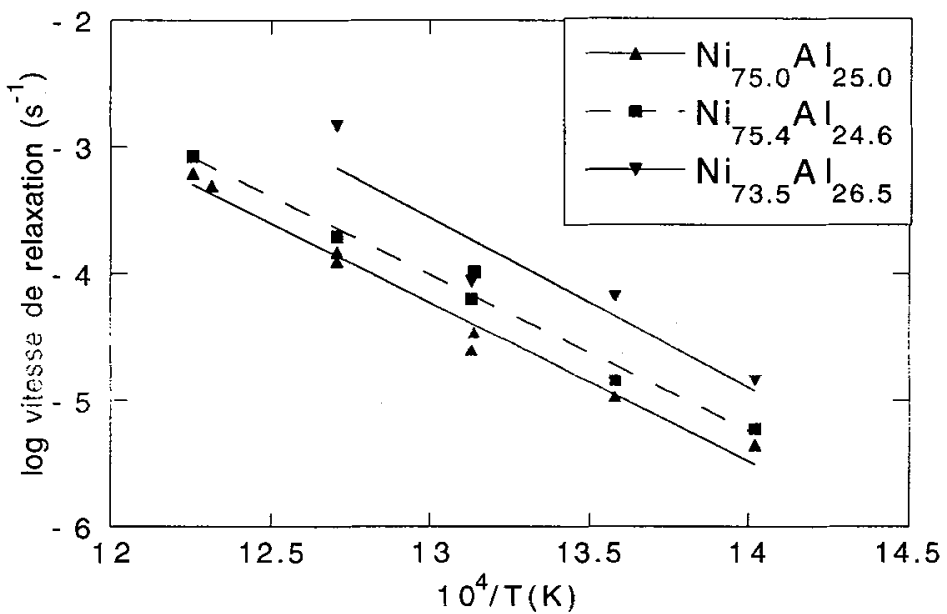

Figure 3. Influence de la température sur la vitesse lente de relaxation de l'ordre atomique dans des composés intermétalliques $\mathrm{Ni}_{3} \mathrm{Al}$ de différentes teneurs en aluminium [4].

Tableau II. Mobilité atomique et propriétés des lacunes dans le nickel, dans des solutions solides et dans des composés intermétalliques basés sur le système $\mathrm{Ni}$-Al $[4,8]$.

\begin{tabular}{|c|c|c|c|}
\hline Matériau & $\begin{array}{c}\text { Enthalpie d'activation } \\
\text { de la mobilité atomique } \\
\qquad \mathrm{HD}(\mathrm{eV})\end{array}$ & $\begin{array}{l}\text { Enthalpie de migration } \\
\text { des lacunes } \\
\mathrm{H}_{\mathrm{m}}(\mathrm{eV})\end{array}$ & $\begin{array}{c}\text { Enthalpie de formation } \\
\text { des lacunes } \\
\mathrm{Hf}_{\mathrm{f}}(\mathrm{eV})\end{array}$ \\
\hline $\mathrm{Ni}$ & 2,88 & $1,04 \pm 0,04$ & $1,8 \pm 0,1$ \\
\hline $\mathrm{Ni}(\mathrm{Al}$ l6) & $2,96 \pm 0,10$ & $1,76 \pm 0,10$ & $1,20 \pm 0,20$ \\
\hline $\mathrm{Ni}(\mathrm{Al} 10)$ & $2,76 \pm 0,07$ & $1,70 \pm 0,06$ & $1,06 \pm 0,13$ \\
\hline $\mathrm{Ni}(\mathrm{Al} 1, \mathrm{Ti} 3)$ & $2,67 \pm 0,24$ & $1,51 \pm 0,09$ & $1,16 \pm 0,33$ \\
\hline $\mathrm{Ni}(\mathrm{Al} 6)$ & $2,96 \pm 0,10$ & $1,76 \pm 0,10$ & $1,20 \pm 0,20$ \\
\hline $\mathrm{Ni}(\mathrm{Al} 4, \mathrm{Ti} 2)$ & $2,79 \pm 0,10$ & $1,96 \pm 0,35$ & $0,83 \pm 0,45$ \\
\hline $\mathrm{Ni}(\mathrm{Al} 4, \mathrm{Ta} 2)$ & $3,06 \pm 0,10$ & $1,94 \pm 0,17$ & $1,12 \pm 0,27$ \\
\hline \multicolumn{4}{|l|}{$\mathrm{Ni} 3 \mathrm{Al}$} \\
\hline 24,6 at $\% \mathrm{Al}$ & $2,88 \pm 0,23$ & $1,38 \pm 0,16$ & $1,50 \pm 0,39$ \\
\hline 25,0 at $\% \mathrm{Al}$ & $2,63 \pm 0,11$ & $1,37 \pm 0,16$ & $1,26 \pm 0,27$ \\
\hline 26,5 at $\% \mathrm{Al}$ & $2,78 \pm 0,16$ & $1,32 \pm 0,16$ & $1,46 \pm 0,32$ \\
\hline $\mathrm{Ni} 3 \mathrm{Al}$ & $2,63 \pm 0,11$ & $1,37 \pm 0,16$ & $1,26 \pm 0,27$ \\
\hline $\mathrm{Ni} 3(\mathrm{Al}, \mathrm{Ti})$ & $3,0 \pm 0,10$ & $1,42 \pm 0,13$ & $1,58 \pm 0,23$ \\
\hline $\mathrm{Ni} 3(\mathrm{Al}, \mathrm{Ta})$ & $2,9 \pm 0,10$ & $1,44 \pm 0,14$ & $1,46 \pm 0,24$ \\
\hline
\end{tabular}




\subsection{Propriétés des lacunes}

Les mesures de la mobilité des lacunes (caractérisée par leur enthalpie de migration) montrent que ces défauts sont moins mobiles dans les solutions solides $\gamma \mathrm{Ni}(\mathrm{Al})$ que dans les composés intermétalliques $\gamma^{\prime} \mathrm{Ni}_{3} \mathrm{Al}$ (Tableau II).

Les additions de Ti ou de Ta ont un effet ralentisseur sur la migration des lacunes dans les deux types de phases $[8,16]$.

Les enthalpies de formation des lacunes ( qui régissent leur concentration d'équilibre) ont été obtenues par différence entre l'enthalpie d'autodiffusion et l'enthalpie de migration. De ce fait, elles comportent une incertitude plus grande. Il apparaît cependant clairement que l'enthalpie de formation des lacunes dans les phases $\gamma$ est nettement plus faible que dans les phases $\gamma$ [8, 17]. A une termpérature donnéc, la concentration de lacunes est donc beatucoup plus importante dans les solutions solides $\gamma$.

C'est ce facteur qui explique la mobilité atomique plus forte trouvée dans les phases $\gamma$ (paragraphe 4.1).

\subsection{Quelques conséquences des résultats expérimentaux}

\section{- Applications de ces résultats à d'autres études fondamentales.}

\section{- Etudes de l'ordre atomique}

Dans la caractérisation structurale de l'ordre atomique par des méthodes de radiocristallographie aux rayons $\mathrm{X}$ ou aux neutrons, il est en général souhaitable de travailler sur des structures d'équilibre. Les résultats présentés ci-dessus permettent de déterminer quelle est la durée du traitement thermique qu'il faut mettre en oeuvre pour obtenir un état d'ordre d'équilibre.

- Etudes de déformation plastique.

Les propriétés mécaniques et les mécanismes de déformation plastique sont influencés par l'état d'ordre existant dans le matériau étudié. De ce point de vue, trois domaines de température sont à considérer :

- aux "basses" températures $\left(\mathrm{T}<\mathrm{T}_{1}\right)$, la mobilité atomique est faible et l'ordre atomique n'évolue pas thermiquement au cours de l'essai. Par contre, il est évidemment modifié par la déformation plastique ellemême.

- aux "hautes" températures ( $\left.\mathrm{T}>\mathrm{T}_{2}\right)$, la mobilité atomique est suffisante pour que l'ordre atomique s'ajuste instantanément à sa valeur d'équilibre à la température $\mathrm{T}$. La déformation se fait à état d'ordre constant.

- les températures "intermédiaires" ( $\left.\mathrm{T}_{1}<\mathrm{T}<\mathrm{T}_{2}\right)$ correspondent à un domaine complexe, où l'effet de l'état d'ordre sur la déformation devrait être maximal et où la vitesse de déformation aura un rôle très important.

Pour situer les idées, le tableau III donne quelques exemples des limites de ces domaines, obtenues en définissant (un peu arbitrairement) pour un essai de déformation classique la température $T_{1}$ comme étant celle qui correspond à un temps de relaxation de l'ordre atomique de 1 heure, et la température $T_{2}$ par un temps de relaxation de 1 seconde. 
Tableau III. Températures correspondant à des temps de relaxtion de l'ordre atomique de 1 heure et de 1 seconde, dans des solutions solides et dans des composés intermétalliques.

\begin{tabular}{|c|c|c|}
\hline Matériau & $\mathrm{T}_{1}\left({ }^{\circ} \mathrm{C}\right)$ pour $\tau=1$ heure & $\mathrm{T}_{2}\left({ }^{\circ} \mathrm{C}\right)$ pour $\tau=1$ seconde \\
\hline $\mathrm{Ni}(\mathrm{Al} 6)$ & 425 & 580 \\
$\mathrm{Ni}(\mathrm{Al} 4, \mathrm{Ti} 2)$ & 450 & 610 \\
\hline $\mathrm{Ni} 3 \mathrm{Al}$ stoechiométrique & 530 & 750 \\
$\mathrm{Ni3}(\mathrm{Al}, \mathrm{Ta})$ & 590 & 790 \\
\hline
\end{tabular}

\section{- Modélisation de comportements macroscopiques.}

Les données quantitatives obtenues (fréquences de saut atomique, enthalpies de formation des lacunes, enthalpies de migration des lacunes) sont celles qu'il est nécessaire d'introduire dans les codes de calcul destinés à modéliser les phénomènes impliquant un transport de matière.

- Signification des mobilités atomiques mesurées.

Les mobilités atomiques, tirées des cinétiques de relaxation de l'ordre atomique dans les alliages, représentent une moyenne sur les fréquences de saut des différents éléments présents. Pour un alliage binaire $A-B$, on montre que le coefficient de diffusion effectif régissant la cinétique de variation de l'ordre, $\mathrm{D}_{\text {eff, est donné par }}$

$$
1 / D_{\text {eff }}=C_{A} / D_{B}{ }^{*}+C_{B} / D_{A}^{*}
$$

où $\mathrm{C}_{\mathrm{A}}$ est la concentration atomique de l'élément $\mathrm{A}$, et $\mathrm{D}_{\mathrm{A}}{ }^{*}$ son coefficient d'auto-diffusion dans l'alliage [18].

C'est le même coefficient de diffusion effectif $D_{\text {eff }}$ qui est pertinent pour décrire la déformation viscoplastique aux températures élevées [19]. Les mesures de mobilité atomique tirées des cinétiques de relaxation de l'ordre sont donc directement utilisables pour modéliser ce type de déformation.

\section{- Effets des éléments d'addition.}

La présence des éléments d'addition titane ou tantale provoque une diminution de la mobilité atomique qui se traduit, aux températures où nos expériences ont été effectuées $\left(400-600^{\circ} \mathrm{C}\right)$, par un décalage $\Delta \mathrm{T}$ des phénomènes vers les hautes températures (de l'ordre de $20^{\circ}$ dans les phases $\gamma$ et de $40-50^{\circ}$ dans les phases $\gamma^{\prime}$ ).

Ce décalage, très appréciable, doit aussi exister aux températures élevées. En effet, pour un même processus de base, défini par son enthalpie d'activation, on s'attend à ce que le rapport $\Delta \mathrm{T} / \mathrm{T}$ soit constant. Ainsi, dans le cas de mécanismes régis par la diffusion, on peut prévoir que les additions de titane ou de tantale induiront, autour de $1000^{\circ} \mathrm{C}$, un décalage des phénomènes vers les hautes températures de $\approx+60$ à $80^{\circ}$ dans les phases intermétalliques.

\section{EXTENSION DE CES METHODES AU COMPOSE INTERMETALLIQUE TIAI}

Les méthodes expérimentales présentées ci-dessus sont également applicables pour caractériser la mobilité atomique et les propriétés des lacunes dans d'autres matériaux pour hautes températures.

La figure 4 illustre ainsi la comparaison de composés intermétalliques Ni3Al et TiAl. Dans un matériau recuit successivement à des températures croissantes, des changements de l'état d'ordre commencent à se produire au dessus d'une température telle que la mobilité atomique devienne appréciable. La figure montre que la température à laquelle une diminution thermique de l'ordre atomique (mise en évidence par une 
augmentation de la résisitivité) commence à intervenir est plus élevée d'environ $80^{\circ}$ dans TiAl que dans $\mathrm{Ni}_{3} \mathrm{Al}$ stœchiométrique. Cette différence est importante puisque des phénomènes tels que le fluage ou l'évolution structurale de matériaux biphasés doivent se produire à température plus élevée dans TiAl que dans Ni3Al. On voit l'intérêt qu'il y aurait à déterminer comment la mobilité atomique varie dans TiAl en fonction de la composition chimique, par exemple : concentration d'aluminium, présence d'éléments d'addition $(\mathrm{Nb}, \mathrm{Cr}$, $\mathrm{Mn}, .$.$) , d'impuretés substitutionelles (\mathrm{Fe}, .$.$) ou interstitielles (\mathrm{O}, .$.$) .$

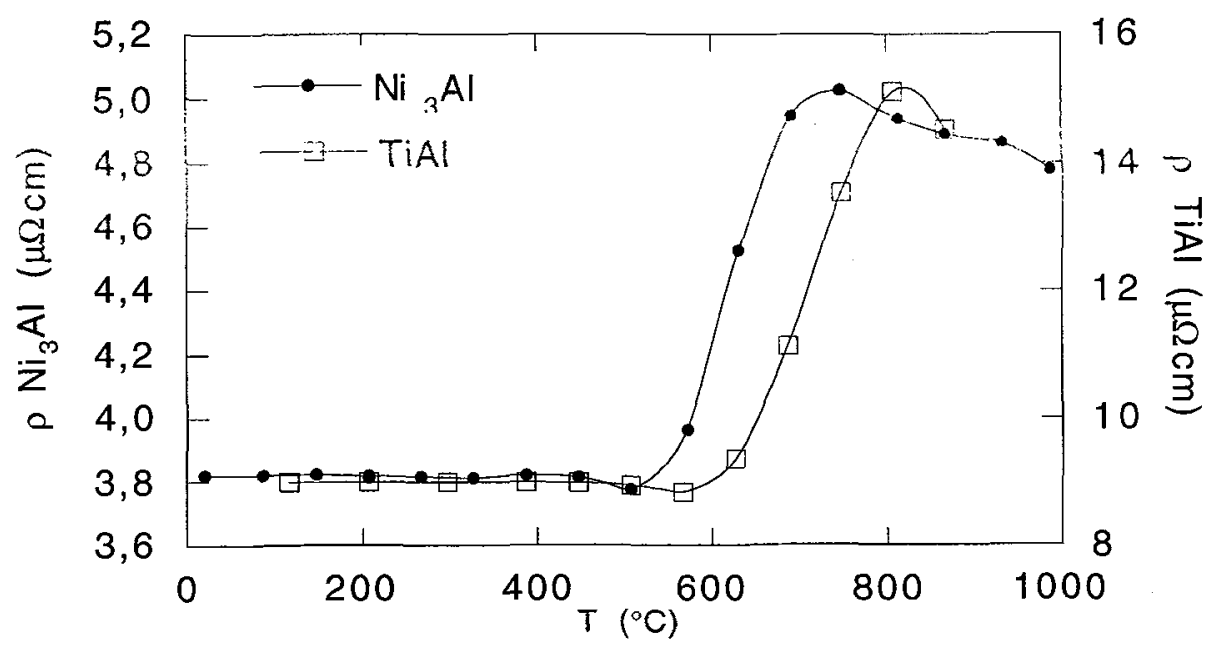

Figure 4. Evolution de la résistivité électrique résiduelle de Ni3 Al et de TiAl stœchimétriques au cours de recuits isochrones à températures croissantes

\section{References}

[1] The role of climb in creep processes

A.K. MUKHERJEE, J.E. BIRD et J.E. DORN

Trans. A.S.M. 62 (1968) 155

[2] The kinetics of precipitation from supersaturated solid solutions

I.M. LIFSCHITZ et V.V. SLYOZOV

J. Phys. Chem. Solids 19 (1961) 35-50

[3] Theorie der Alterung von Niederschlagen durch Umlösen (Ostwald-Reifung)

C. WAGNER

Z. Elektochemie 65 (1961) 581

[4] Kinetics of long-range order relaxation in $\mathrm{Ni}_{3} \mathrm{Al}$ : the effect of stoichiometry

C. DIMITROV, X. ZHANG et O. DIMITROV

Acta metall. mater. $\underline{43}$ (1995) sous presse

[5] Equilibrium and kinetics of thermal ordering or disordering in Ni3Al

C. DIMITROV, T. TARFA et O. DIMITROV

Ordering and Disordering in Alloys, Ed. R. A. Yavari (Elsevier Appl. Sc., London,1992) pp. 130-7 
[6] Equilibrium and kinetics of local ordering in $\mathrm{Ni}(\mathrm{Al})$ solid solutions

B. SITAUD et O. DIMITROV

J. Phys.: Condens. Matter, 2 (1990) 7061-75

[7] A resistometric study of order changes in $\mathrm{Ni}_{3} \mathrm{Al}$ : Composition dependence of equilibrium and kinetics.

C. DIMITROV, X. ZHANG, T. TARFA et O. DIMITROV

Intermetallic Compounds, Ed. O. Izumi (The Japan Institute of Metals, Sendai, 1991) pp.75-79

[8] Mobilité atomique et propriétés des lacunes dans des phases modèles de superalliages à base de nickel.

Effets des additions de titane et de tantale.

T. TARFA

Thèse de Doctorat de l'Université Paris VI, 1994

[9] Displacement threshold energies in $\mathrm{Ni}(\mathrm{Al})$ solid solutions and in $\mathrm{Ni}_{3} \mathrm{Al}$.

C. DIMITROV. B. SITAUD, O. DIMITROV

Journal of Nuclear Materials 208 (1994) 53-60

[10] Radiation-induced defects in solid solutions and intermetallic compounds based on the $\mathrm{Ni}-\mathrm{Al}$ system

1. Low-temperature electron-irradiation damage

C. DIMITROV, B. SITAUD, X. ZHANG, O. DIMITROV, U. DEDEK, and F. DWORSCHAK

J. Phys.: Condens. Matter 4 (1992) 10199-210

[11] Composition dependence of the properties of irradiation-induced defects in $\gamma \mathrm{Ni}(\mathrm{Al}), \gamma \mathrm{Ni}(\mathrm{Al}, \mathrm{Ti})$ and $\gamma$ $\mathrm{Ni} 3 \mathrm{Al}$ alloys

C. DIMITROV, X. ZHANG, B. SITAUD, O. DIMITROV, U. DEDEK, F. DWORSCHAK

Advanced Materials and Processes, Eds. H. E. Exner, V. Schumacher, Vol. 1 (DGM, Oberursel,1990) p. 435

[12] Electron-irradiation induced defects in stoichiometric and off-stoichiometric $\mathrm{Ni}_{3} \mathrm{Al}$

B. SITAUD, C. DIMITROV, G. DAI, P. MOSER et O. DIMITROV

Intermetallic Compounds, Ed. O. Izumi (The Japan Institute of Metals, Sendai, 1991) pp.69-73

[13] Radiation-induced defects in solid solutions and intermetallic compounds based on the Ni-Al system

II. Recovery of radiation damage

C. DIMITROV, B. SITAUD, X. ZHANG, O. DIMITROV, U. DEDEK, and F. DWORSCHAK

J. Phys.: Condens. Matter 4 (1992) 10211-26

[14] Influence of structure and composition on atomic mobility in $\mathrm{Ni}\left(\mathrm{Al}\right.$ )-based solid solutions and $\mathrm{Ni}_{3} \mathrm{AI}$ based intermetallics.

T. TARFA, C. DIMITROV, O. DIMITROV

Journal de Physique IV, Colloque C7, supp. Journal de Physique III, Volume 3 (nov. 1993) 453-6.

[15] The effects of Ti or Ta on the kinetics of short-range ordering and self-diffusion in Ni(Al)-based solid solutions.

T. TARFA, B. SITAUD, O. DIMITROV

Acta metall. mater. 41 (1993) 3191-202.

[16] Irradiation-induced defects in solid solutions and intermetallic compounds of the $\mathrm{Ni}-\mathrm{Al}$ system: the effects of a third element ( $\mathrm{Ti}$ or $\mathrm{Ta}$ ).

T. TARFA, O. DIMITROV

Journal de Physique IV, Colloque C7, supp. Journal de Physique III, Volume 3 (nov. 1993) 449-52.

[17] Autodiffusion et propriétés des lacunes dans des solutions solides à base de nickel, modèles de la phase $\gamma$ des superalliages.

B. SITAUD

Thèse de Doctorat de l'Université Paris VI, 1991

[18] Energies de formation et de migration des lacunes dans les alliages fer-nickel de structure c.f.c. par la méthode de l'anisotropie magnétique induite

A. CAPLAIN, W. CHAMBRON

Acta metall. 25 (1977) 1001-11

[19] Effective diffusion coefficient involved in the high temperature viscoplastic behaviour law of Cu-Sn (15-

20 at $\% \mathrm{Sn}$ )

G. SOME, J. BERNARDINI, C. LEXCELLENT

Acta metall. mater. $\underline{40}$ (1991) 761-9 